\title{
Acolhimento no cuidado odontológico: revisão integrativa
}

\author{
User embracement in dental care: integrative review
}

Acogimiento en la práctica odontológica: revisión integradora

Semíremis Silva Santos ${ }^{1}$, Marcondes Pantaleão de Amorim ${ }^{1}$, Lidianne Mércia Barbosa Malta Rocha ${ }^{1}$, Ana Maria Guerra Costa ${ }^{1}$, Lilian Maria Santos Silva de Lira ${ }^{1}$, Angélica Patricia León Gaines ${ }^{2}$, Ellen Karla Nobre dos Santos-Lima ${ }^{1 *}$.

\section{RESUMO}

Objetivo: Revisar sobre o acolhimento na humanização do cuidado odontológico. Métodos: Trata-se de uma revisão integrativa da literatura realizada em artigos disponíveis nas bases de dados online da Biblioteca Virtual em Saúde (BVS), PubMed e PubMed Central (PMC) publicados até janeiro de 2022. Os descritores utilizados foram acolhimento e Odontologia em combinação através da estratégia "acolhimento AND Odontologia". Foram incluídos artigos publicados em texto completo (free full text) de 2016 a 2021, nos idiomas português ou inglês. Resultados: Na literatura analisada, observou-se o acolhimento como fator primordial para promover a humanização no cuidado odontológico. As ferramentas sugeridas para facilitar o acolhimento foram: humanização da assistência em clínica escola, bom relacionamento interpessoal entre os profissionais e com o paciente, conhecimento e ruptura de preconceitos através da vivência clínica com pacientes com necessidades especiais, visita domiciliar, associação de ambiência, tranquilidade e confiança; agendamento de consultas odontológicas e rede social. Considerações finais: $O$ acolhimento possui importância crescente no atendimento humanizado, na construção de uma relação de confiança com o paciente, contribuindo para o sucesso do tratamento. É importante oferecer um tratamento humanizado com visão social, boa comunicação e acolhimento. Dessa maneira, os pacientes serão mais colaborativos e os tratamentos terão mais respostas positivas.

Palavras-chave: Humanização da assistência, Odontologia, Acolhimento, Educação em odontologia.

\section{ABSTRACT}

Objective: To review about the user embracement in the humanized dental care. Methods: The integrative literature review was carried out on articles available in the online database of the Biblioteca Virtual em Saúde (BVS), PubMed and PubMed Central (PMC) published until January 2022. The uniterms "user embracement" and "Dentistry" were used in combination through the "user embracement AND Dentistry" strategy. Articles published in free full text from 2016 to 2021 in Portuguese or in English were included. Results: In the literature analyzed, embracement was observed as a key factor to promote humanized dental care. The tools suggested to facilitate the user embracement were humanization of care in clinical schools, good interpersonal relationships between professionals and with the patient, knowledge and rupture of prejudices through clinical experience with patients with special needs, homecare, association of ambience, tranquility and trust; scheduling dental appointments and social network site. Final considerations: User embracement has increasingly importance in humanized care, in building a relationship of trust with the patient, contributing to the success of treatment. It is important to offer a humanized treatment with a social vision, good communication and user embracement. In this way, patients will be more collaborative and treatments will have more positive responses.

Keywords: Humanization of assistance, Dentistry, User embracement, Dental education.

${ }^{1}$ Centro Universitário Mario Pontes Jucá (UMJ), Maceió - AL. *E-mail: ellen.nobre@umj.edu.br

${ }^{2}$ Facultade da Saude (FUSM), Bogota, Colômbia. 


\section{RESUMEN}

Objetivo: Revisar sobre acogimiento de los pacientes en la humanización del entorno odontológico. Metodos: La revisión bibliográfica integradora se realizó sobre artículos disponibles en la base de datos online de la Biblioteca Virtual de Salud (BVS), PubMed y PubMed Central (PMC) publicados hasta enero de 2022. Los descriptores utilizados fueron acogimiento y Odontología en combinación a través de la estrategia "acolhimento AND Odontologia". Se incluyeron los artículos publicados a texto completo (free full text) entre 2016 y 2021, en los idiomas portugués e inglés. Resultados: En la literatura analizada, la acogida se observó como un factor clave para promover la humanización en la atención odontológica. Las herramientas sugeridas para facilitar la acogida fueron: humanización del cuidado en las practicas clínicas del pregrado, buena relación interpersonal entre profesionales y con el paciente, conocimiento y ruptura de prejuicios a través de la experiencia clínica con pacientes con necesidades especiales, visitas domiciliarias, asociación de ambiente, tranquilidad y confianza; programación de citas dentales y red social. Consideraciones finales: La acogida es cada vez más importante en el cuidado humanizado, en la construcción de una relación de confianza con el paciente, contribuyendo al éxito del tratamiento. Es importante ofrecer un trato humano con visión social, buena comunicación y acogida. De esta forma, los pacientes serán más colaborativos y los tratamientos tendrán respuestas más positivas.

Palabras clave: Humanización de la atención, Odontología, Acogimiento, Educación en odontología.

\section{INTRODUÇÃO}

A temática da humanização vem sendo cada vem mais debatida, de modo a abordar os conhecimentos, práticas e atitudes dos profissionais da área de saúde, cujo objetivo principal deve ser proporcionar qualidade nas consultas. Nesse contexto, o processo de atendimento humanizado deve ser centrado em procedimentos que visem beneficiar o usuário, proporcionando privacidade, autonomia e evitando intervenções desnecessárias (MINISTÉRIO DA SAÚDE, 2010; MINISTÉRIO DA SAÚDE, 2013).

De acordo com o Descritores em Ciências da Saúde (DeCS), o acolhimento representa a organização do fluxo de trabalho para atender os indivíduos que procuram os serviços de saúde, com o objetivo de fortalecer os princípios de universalidade, integralidade e equidade. Tal reorganização estimula e promove reflexões e ações de Humanização dos Serviços de Saúde, com fundamento na ética e na cidadania (ID do descritor: DDCS050296).

As relações de cuidado dependem de quem participa dos processos e de como e em que condições o processo ocorre. O processo de acolhimento possibilita a humanização do cuidado, com coordenação e vinculação efetiva das relações entre profissionais e usuários. Tal processo ocorre através de escuta qualificada e formação de vínculo e respeito, cujo foco é a compreensão e a resolução do problema abordado pelo paciente. Portanto, compreende-se que o acolhimento aproxima e humaniza as relações entre trabalhadores dos serviços de saúde com seus usuários (LOPES GVDO, et al., 2014).

A prática do acolhimento deve ser realizada em todas as relações do cuidado, adaptando-se aos participantes e às condições dos processos. $O$ acolhimento em saúde está alicerçado em três pilares: postura profissional, atitude e tecnologia do cuidado. Por sua vez, tal tecnologia diz respeito ao mecanismo de ampliação e facilitação do acesso, bem como à organização do processo de trabalho em equipe (MINISTÉRIO DA SAÚDE, 2013; LOPES AS, et al., 2015; DARTIGUELONGUE JB e CAFIERO PJ, 2021).

Para Mitre SM, et al. (2012), a relação entre os usuários e os profissionais em saúde deve ter o reconhecimento das necessidades como um norte para o atendimento de qualidade, em que há acolhimento do outro; compreensão e significação de suas singularidades; e oferta dos saberes em saúde. Tais abordagens permitem intervenções continuadas de vínculo, produção de autonomia e responsabilização do usuário pelo resultado das intervenções. $O$ acolhimento aproxima e humaniza as relações entre trabalhadores e serviços de saúde com seus usuários através de escuta qualificada e de formação de vínculo e respeito; favorecendo a resposta positiva e a responsabilização do usuário pela solução do seu problema. 
Portanto, uma assistência humanizada voltada ao acolhimento atende as necessidades dos usuários de maneira qualificada. Esse contato entre os profissionais e os pacientes permite a criação responsável de um serviço resolutivo, possibilita solução do problema e encaminhamento assertivo (LOPES AS, et al., 2015).

$\mathrm{Na}$ Odontologia, os pacientes estão, progressivamente, mais informados e exigentes sobre os diversos tipos de tratamento. É importante personalizar, entender cada caso, respeitando as decisões individuais. Cada ser humano é único e traz consigo seus conceitos, medos e traumas. Então o profissional que considera esses fatores pode construir o sucesso de qualquer tratamento (SCHNEIDER A, et al., 2018).

Dessa forma, o presente trabalho objetivou realizar uma revisão integrativa da literatura sobre 0 acolhimento no ambiente odontológico buscando ferramentas e condutas utilizadas no acolhimento em serviço de saúde odontológico.

\section{MÉTODOS}

A revisão integrativa da literatura foi realizada em artigos publicados até janeiro de 2022, disponíveis nas bases de dados online Biblioteca Virtual em Saúde (BVS), PubMed e PubMed Central (PMC). A BVS inclui as bases Bibliografia Brasileira de Odontologia (BBO), Scientific Electronic Library Online (SciELO), Literatura Latino-Americana e do Caribe em Ciências da Saúde (LILACS), MEDLINE e Cochrane.

Os descritores utilizados para a busca foram: acolhimento e Odontologia (user embracement AND Dentistry). Os critérios de inclusão foram: texto completo, idioma português ou inglês, publicação realizada nos últimos 5 anos e assunto principal "acolhimento" e "educação em Odontologia". Os critérios de exclusão foram artigo duplicado, artigo em outra temática e ausência de clareza quanto ao assunto principal "acolhimento".

O Quadro 1 traz o detalhamento das quatro etapas desta revisão integrativa, desde o tema escolhido até a classificação dos trabalhos incluídos, por modelo de estudo.

Quadro 1 - Detalhamento das etapas da revisão integrativa

\begin{tabular}{|c|c|c|}
\hline Etapa & Tópicos & Detalhamento \\
\hline \multirow{6}{*}{$1^{\mathrm{a}}$} & Tema & Acolhimento na Odontologia \\
\hline & Pergunta norteadora & $\begin{array}{l}\text { Quais são as ferramentas e condutas utilizadas na } \\
\text { implantação da humanização no serviço de saúde } \\
\text { odontológico? }\end{array}$ \\
\hline & Objetivo & $\begin{array}{l}\text { Realizar uma revisão integrativa da literatura sobre o } \\
\text { acolhimento no ambiente odontológico. }\end{array}$ \\
\hline & Estratégia de busca & $\begin{array}{l}\text { Acolhimento AND Odontologia } \\
\text { (User embracement AND Dentistry) }\end{array}$ \\
\hline & Descritores DeCS / MeSH & Acolhimento; Odontologia \\
\hline & Bibliotecas virtuais & $\begin{array}{l}\text { Biblioteca Virtual em Saúde (BVS) (inclui as bases BBO, } \\
\text { SciELO, LILACS, MEDLINE, Cochrane); PubMed; } \\
\text { PubMed Central (PMC) }\end{array}$ \\
\hline \multirow{3}{*}{$2^{\mathrm{a}}$} & Período de coleta dos dados & Setembro de 2021 a janeiro de 2022 \\
\hline & Critérios de inclusão & $\begin{array}{l}\text { Texto completo; Data da publicação (2016 - 2022); Idioma } \\
\text { português ou inglês; Assunto principal "Acolhimento" e } \\
\text { "Educação em Odontologia" }\end{array}$ \\
\hline & Critérios de exclusão & $\begin{array}{l}\text { Artigo duplicado; Artigo em outra temática; Ausência de } \\
\text { clareza quanto ao assunto principal "acolhimento" }\end{array}$ \\
\hline $3^{\mathrm{a}}$ & $\begin{array}{l}\text { Número de trabalhos } \\
\text { selecionados para avaliação }\end{array}$ & 7 publicações científicas \\
\hline $4^{\mathrm{a}}$ & $\begin{array}{l}\text { Categorias obtidas com a análise } \\
\text { dos trabalhos investigados. }\end{array}$ & $\begin{array}{l}\text { Estudo qualitativo (3); Revisão integrativa (1); Dissertação } \\
\text { (1); Estudo descritivo (1); Estudo transversal (1) }\end{array}$ \\
\hline
\end{tabular}

Fonte: Silva S, et al., 2022. 


\section{RESULTADOS}

O fluxograma traz as etapas da seleção dos trabalhos para a revisão integrativa na base BVS, detalhando as etapas de identificação, triagem, elegibilidade e inclusão dos trabalhos pesquisados. Dos 23 trabalhos encontrados na BVS, publicados de 2016 a 2021, cinco foram incluídos nesta revisão e analisados para identificação de ferramentas e condutas utilizadas para o acolhimento (Figura 1).

Figura 1 - Etapas da seleção dos trabalhos para a revisão integrativa

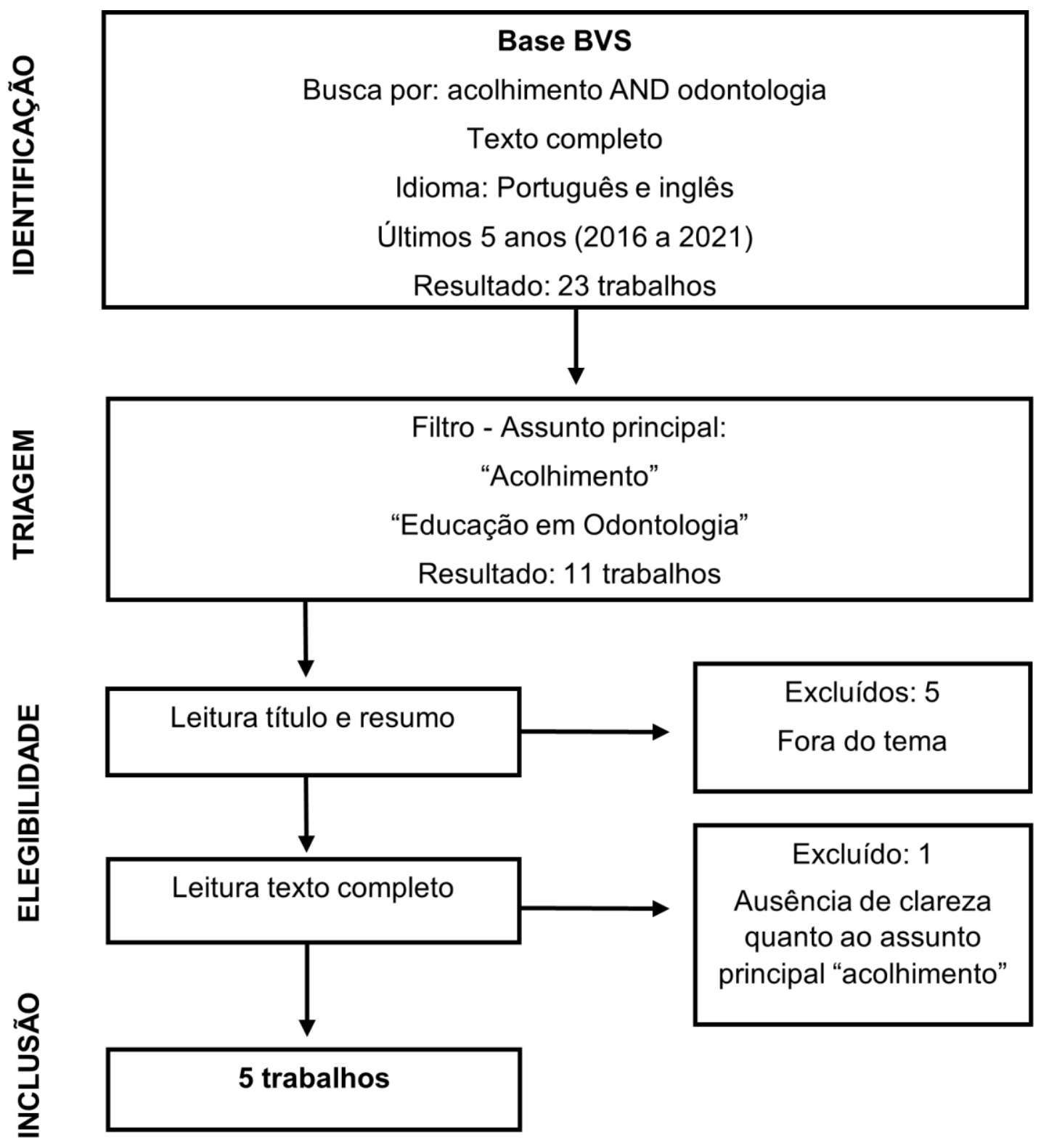

Fonte: Silva S, et al., 2022.

A base PubMed apresentou dois resultados, sendo um artigo dentro da temática do presente estudo. A base PubMed Central (PMC) também apresentou dois resultados não redundantes, dos quais um estava dentro da temática. O Quadro 2 resume as principais informações extraídas do total de sete publicações incluídas no estudo. 
Quadro 2 - Ferramentas e condutas utilizadas para o acolhimento de acordo com as publicações incluídas no estudo.

\begin{tabular}{|c|c|c|c|c|}
\hline Título & Referência & $\begin{array}{l}\text { Tipo de } \\
\text { estudo }\end{array}$ & Ferramenta & Conduta \\
\hline $\begin{array}{l}\text { Humanização: } \\
\text { fragilidades, desafios e } \\
\text { fortalezas em uma } \\
\text { escola de Odontologia. }\end{array}$ & $\begin{array}{l}\text { Rodrigues } \\
\text { MP, et al. } \\
(2016)\end{array}$ & $\begin{array}{l}\text { Estudo } \\
\text { qualitativo. }\end{array}$ & $\begin{array}{l}\text { Humanização da } \\
\text { assistência em clínica } \\
\text { escola. }\end{array}$ & $\begin{array}{l}\text { Promoção de maior } \\
\text { integração entre o corpo } \\
\text { docente, acadêmicos e } \\
\text { usuários das clínicas } \\
\text { das IES (valorização do } \\
\text { respeito mútuo). }\end{array}$ \\
\hline $\begin{array}{l}\text { A visita domiciliar } \\
\text { como prática } \\
\text { pedagógica na } \\
\text { formação em } \\
\text { Odontologia. }\end{array}$ & $\begin{array}{l}\text { Silva RM, } \\
\text { et al. } \\
\text { (2017). }\end{array}$ & $\begin{array}{l}\text { Revisão } \\
\text { integrativo. }\end{array}$ & Visita domiciliar (VD). & $\begin{array}{l}\text { Ampliação do acesso } \\
\text { aos serviços e criação } \\
\text { de vínculo com } \\
\text { a população. }\end{array}$ \\
\hline $\begin{array}{l}\text { Humanização em } \\
\text { saúde: a vivência em } \\
\text { uma instituição de } \\
\text { ensino. }\end{array}$ & $\begin{array}{l}\text { Silva RRD } \\
\text { (2018). }\end{array}$ & Dissertação. & $\begin{array}{c}\text { Bom relacionamento } \\
\text { interpessoal entre os } \\
\text { profissionais e com o } \\
\text { paciente. }\end{array}$ & $\begin{array}{l}\text { Adequação da } \\
\text { linguagem técnica para } \\
\text { a melhor compreensão } \\
\text { pelo paciente durante as } \\
\text { orientações (gera } \\
\text { motivação e adesão ao } \\
\text { tratamento). }\end{array}$ \\
\hline $\begin{array}{c}\text { Saúde bucal e } \\
\text { Pacientes com } \\
\text { Necessidades } \\
\text { Especiais (PNE): } \\
\text { percepções de } \\
\text { graduandos em } \\
\text { Odontologia da FOB- } \\
\text { USP. }\end{array}$ & $\begin{array}{l}\text { Jacomine } \\
\text { JC, et al. } \\
\text { (2018). }\end{array}$ & $\begin{array}{c}\text { Estudo } \\
\text { qualitativo. }\end{array}$ & $\begin{array}{l}\text { Conhecimento e } \\
\text { ruptura de preconceitos } \\
\text { através da vivência } \\
\text { clínica com PNE. }\end{array}$ & $\begin{array}{l}\text { Capacitação dos } \\
\text { graduandos para o } \\
\text { acolhimento do paciente } \\
\text { com necessidades } \\
\text { especiais. }\end{array}$ \\
\hline $\begin{array}{l}\text { Compreensão sobre o } \\
\text { atendimento } \\
\text { humanizado em um } \\
\text { ambulatório de } \\
\text { Odontologia da } \\
\text { marinha. }\end{array}$ & $\begin{array}{l}\text { Silva KAR, } \\
\text { Dias AA } \\
\text { (2019). }\end{array}$ & $\begin{array}{l}\text { Estudo } \\
\text { qualitativo. }\end{array}$ & $\begin{array}{l}\text { Associação das } \\
\text { ferramentas ambiência, } \\
\text { tranquilidade, } \\
\text { confiança ao } \\
\text { acolhimento. }\end{array}$ & $\begin{array}{l}\text { Escuta cuidadosa } \\
\text { dos pacientes. }\end{array}$ \\
\hline $\begin{array}{l}\text { Look who's talking } \\
\text { now: Cancer in } \\
\text { primary care on } \\
\text { Twitter. An } \\
\text { observational study. }\end{array}$ & $\begin{array}{l}\text { Milley KM, } \\
\text { et al. } \\
(2021)\end{array}$ & $\begin{array}{c}\text { Estudo } \\
\text { descritivo. }\end{array}$ & Rede social. & \begin{tabular}{lr}
\multicolumn{2}{l}{ Comunicação (diálogo) } \\
de informações \\
educativas \\
profissionais pelos \\
organizações de saúde.
\end{tabular} \\
\hline $\begin{array}{l}\text { Indicators for dental } \\
\text { appointment } \\
\text { scheduling in primary } \\
\text { health care: a national } \\
\text { cross-sectional study. }\end{array}$ & $\begin{array}{l}\text { Melo EA, } \\
\text { et al. } \\
(2021)\end{array}$ & $\begin{array}{l}\text { Estudo } \\
\text { transversal. }\end{array}$ & $\begin{array}{l}\text { Agendamento de } \\
\text { consultas } \\
\text { odontológicas. }\end{array}$ & $\begin{array}{l}\text { Ampliação do acesso ao } \\
\text { serviço odontológico. }\end{array}$ \\
\hline
\end{tabular}

Fonte: Silva S, et al., 2022.

As ferramentas sugeridas para facilitar o acolhimento foram: humanização da assistência em clínica escola, bom relacionamento interpessoal entre os profissionais e com o paciente, conhecimento e ruptura de preconceitos através da vivência clínica com pacientes com necessidades especiais, visita domiciliar, associação de ambiência, tranquilidade e confiança; agendamento de consultas odontológicas e rede social (RODRIGUES MP, et al., 2016; SILVA RM, et al., 2017; SILVA RR, et al., 2018; JACOMINE JC, et al., 2018; SILVA KA e DIAS AA, et al., 2019; MILLEY KM, et al., 2021; MELO EA, et al., 2021). 
Segundo as publicações analisadas, em ordem cronológica, observa-se que a humanização inicia na relação interpessoal acadêmica. Rodrigues MP, et al. (2016) apontam que as relações conflituosas entre docentes e discentes têm efeitos negativos na formação do acadêmico, destacando a importância da implantação e da manutenção de um ambiente uníssono, harmônico, tranquilo e seguro de orientação acadêmica.

Silva RM, et al. (2017) observaram que a prática (ferramenta) da visita domiciliar contribuiu para um novo agir pedagógico como um cenário propício para que o estudante de Odontologia se aproximasse dos usuários do território e dos demais profissionais da equipe de saúde, indo ao encontro do preconizado pelas Diretrizes Curriculares Nacionais para Cursos de Graduação em Odontologia.

Ao passo que Jacomine JC, et al. (2018), ao analisarem as percepções de graduandos em Odontologia em um estudo qualitativo, afirmam que existe uma parcela significativa de estudantes de Odontologia que não se sentem preparados para o atendimento a pacientes com necessidades especiais (PNE) e ressaltam a extrema importância do contato dos PNE com os graduandos para que os estudantes quebrem possíveis preconceitos e assim promovam saúde bucal e qualidade de vida aos PNE. Para isso, faz-se necessária a capacitação dos graduandos para o acolhimento do paciente com necessidades especiais.

O trabalho de Silva RRD (2018) reporta que ainda existem dificuldades em trabalhar a humanização no Ensino Superior, reiterando a necessidade de se trabalhar este tema em todo o percurso da graduação. Destaca a importância do bom relacionamento interpessoal entre os profissionais e com o paciente e orienta que haja adequação da linguagem técnica para a melhor compreensão pelo paciente durante as orientações, o que gera motivação e favorece a adesão ao tratamento.

Nesse contexto do atendimento humanizado, Silva KAR e Dias AA, et al. (2019) buscam compreender a ambiência e o acolhimento preconizados na Política Nacional de Humanização sob a ótica dos cirurgiõesdentistas e dos usuários de um atendimento ambulatorial odontológico. E, em estudo qualitativo, ressaltam a importância de haver ambiência, tranquilidade, confiança, acolhimento e escuta cuidadosa dos pacientes em um ambulatório de Odontologia da marinha.

Segundo o estudo publicado por Melo EA, et al. (2021), fatores associados ao agendamento de consultas odontológicas podem afetar positiva ou negativamente a utilização dos serviços odontológicos. Tal estudo avaliou os indicadores de agendamento de consultas odontológicas na Atenção Básica à Saúde e observaram que $58,1 \%$ dos usuários entrevistados nas Unidades Básicas de Saúde (UBS) procuram o atendimento odontológico disponível. Em relação à idade, pacientes com até 42 anos têm maior chance de procurar o dentista. Os resultados sugerem que quando as equipes de saúde bucal colocam em prática as diretrizes da Política Nacional de Saúde Bucal, atendendo apenas uma equipe de saúde da família, a chance de os usuários da UBS buscarem consulta odontológica é maior do que quando as equipes de saúde bucal atendem mais de uma equipe de saúde da família.

Diante da utilização crescente das redes sociais para circulação de informações na área da saúde, o estudo conduzido por Milley KM, et al. (2021) explorou o tipo de usuário e as informações compartilhadas no Twitter sobre o câncer na atenção primária, a qual é "porta de entrada" do serviço de saúde, na qual os usuários são acolhidos. Os autores concluem que uma ampla gama de usuários utiliza o Twitter para compartilhar informações sobre o câncer na atenção primária. O conteúdo é diferente entre os grupos de usuários, mas o atraso no diagnóstico e a percepção dos sintomas são temas comuns. Os consumidores publicaram mais tweets negativos, principalmente em relação ao atraso no diagnóstico. As organizações de saúde se concentraram na conscientização e no rastreamento dos sintomas. Mais da metade dos tweets da mídia eram sobre diagnóstico ou triagem atrasada (MILLEY KM, et al., 2021).

\section{DISCUSSÃO}

O presente trabalho analisou o acolhimento na relação dentista / paciente e o processo de humanização do serviço prestado. Há carência de publicações que respondam à pergunta norteadora do estudo. Contudo, na literatura analisada, observou-se o acolhimento como fator primordial para promover a humanização do cuidado odontológico. 
A temática da humanização é cada vem mais debatida e aborda conhecimentos, práticas e atitudes que objetivam melhorar a qualidade no atendimento. No Brasil, dentro da Política Nacional de Humanização (PNH), há a Política Nacional de Humanização da Atenção e Gestão no Sistema Único de Saúde (Humaniza SUS), a qual considera que a humanização é a valorização dos diferentes sujeitos que atuam no processo de produção de saúde: usuários, trabalhadores e gestores (MINISTÉRIO DA SAÚDE, 2010).

Os valores que norteiam a Humaniza SUS são: A) autonomia e protagonismo dos sujeitos, B) corresponsabilidade entre os sujeitos, C) estabelecimento de vínculos solidários, D) construção de redes de cooperação e E) participação coletiva no processo de gestão. Tal política demonstra que existe, no Brasil, uma preocupação em torno da humanização nos serviços de saúde, valorizando o vínculo entre trabalhadores de saúde e usuários (MINISTÉRIO DA SAÚDE, 2010).

Lopes GVDO, et al. (2014) afirmam que o processo de acolhimento possibilita a humanização do cuidado, facilitando os procedimentos nos serviços de saúde, de forma segura para a resolução dos problemas, com vinculação efetiva das relações entre profissionais e usuários. Já para Costa G, et al. (2014), a humanização das relações entre trabalhadores e serviços de saúde com os usuários ocorre através do acolhimento. Segundo os autores, deve-se estabelecer como prioridade a necessidade do paciente e a possibilidade de uma construção de uma relação profissional com o paciente através do acolhimento, compartilhando responsabilidades na intervenção resolutiva.

Porém, a humanização esbarra na burocratização do fluxo de atendimento, perdendo-se o vínculo na relação com os pacientes. Por isso, para implantação de ações na atenção básica, a organização do acolhimento é fundamental para ampliar o acesso à atenção básica e aos demais níveis do sistema. Com a organização do acolhimento, seria possível eliminar as filas e conduzir o atendimento com base em riscos priorizados (MINISTÉRIO DA SAÚDE, 2010; MELO EA, et al., 2021).

O acolhimento precisa ser construído por toda a equipe, necessita da dedicação de cada profissional. $O$ entendimento da equipe de saúde envolvida é fundamental nesta relação de acolhimento e humanização, em todas as etapas da atenção. Por isso, é importante que esses personagens sejam acessíveis e possuam um olhar amplo e, sobretudo, que se faça reinar a harmonia no ambiente de saúde (DARTIGUELONGUE JB e CAFIERO PJ, 2021).

Para o acolhimento na demanda espontânea, deve haver mudanças nos modos de organização das equipes, nas relações entre os trabalhadores e nos modos de cuidar. Deve-se acolher com equidade e qualidade, compreendendo a dificuldade do usuário, procurando sempre estabelecer um contato cordial com o paciente a fim de possibilitar um ambiente favorável para resolução de possíveis dificuldades nos procedimentos. Em consonância, no atendimento em um ambulatório de Odontologia, deve haver ambiência, tranquilidade, confiança, acolhimento e escuta cuidadosa dos pacientes (MINISTÉRIO DA SAÚDE, 2013; SILVA KAR e DIAS AA, 2019).

A prática do acolhimento é necessária para o estabelecimento do vínculo. Através do vínculo, na rede psicossocial, experiências são compartilhadas e relações são estabelecidas, inclusive, na instituição de saúde, em que a aproximação entre paciente e profissional de saúde promove um encontro, em que os sujeitos estão em situação de desequilíbrio, havendo habilidades e expectativas diferentes. O paciente busca assistência, em estado físico e emocional fragilizados. E o profissional é capacitado para cuidar da causa da fragilidade. O vínculo criado precisa gerar uma ligação ética entre ambos, numa convivência de ajuda e respeito mútuos (MINISTÉRIO DA SAÚDE, 2010; LOPES GVDO, et al., 2014).

Bem como observaram Silva RM, et al. (2017), a visita domiciliar contribuiu para o estudante de Odontologia se aproximar dos usuários do território e dos demais profissionais da equipe de saúde. Portanto, as dificuldades referentes ao estabelecimento do vínculo podem ser ultrapassadas por meio de aproximação do profissional com a realidade vivenciada pelo usuário, bem como, consequentemente, da construção de relação de confiança, assim, é fundamental conhecer a realidade das pessoas de sua área de trabalho e estabelecer com elas uma verdadeira aproximação (SILVA RM, et al., 2017). 
Contudo, como apontado por Furlin N (2020), a sobrevalorização do tecnicismo na profissão do cirurgiãodentista não é apenas por estudantes, mas também pelas pessoas que acessam ao serviço especializado de saúde bucal. Observa-se que a humanização ainda é pouco vivenciada no processo de formação do profissional, havendo a priorização da formação técnica dos cursos de graduação em Odontologia em detrimento da formação humanística. Realidade que pode ser transformada através do processo de educação tanto do profissional quanto da população (FURLIN N, 2020).

A consulta odontológica possui o componente emocional que possibilita a criação de uma relação íntima e invasiva. Portanto, vale investir na conscientização do paciente de que o trabalho do cirurgião-dentista vai além de um caráter estético e de alívio da dor. Além disso, o paciente que é tratado com atenção torna-se mais cooperativo durante o procedimento clínico. O dentista pode usar a empatia ao seu favor, estabelecendo uma relação que poderá minimizar e até suprimir a ansiedade do paciente, criando um ponto de confiança, segurança, tranquilidade e serenidade, as quais devem ser estimuladas pelo dentista durante o cuidado odontológico (MOTA L, et al., 2013).

Segundo Cianetti S, et al. (2017), desde a infância, ansiedade e medo do procedimento odontológico precisam ser prevenidos e trabalhados. Pois "medo / ansiedade de dentista" é um problema comum em crianças / adolescentes em todo o mundo. Novas estratégias para superar essa relevante condição infantojuvenil devem ser estimuladas. Silveira ER, et al. (2021) conduziram uma metanálise, registrando que o "medo de dentista" é prevalente também em adultos em todo o mundo, sendo mais prevalentes entre as mulheres. As evidências sugerem que o "medo de dentista" afeta negativamente o padrão de atendimento odontológico e as condições clínicas e subjetivas de saúde bucal. Um melhor conhecimento da prevalência global e dos fatores associados a este problema pode justificar estratégias de prevenção que podem modular 0 comportamento do indivíduo em consultório odontológico.

Mota L, et al. (2013) entendem que o ambiente odontológico está carregado de sentimentos de aversão em razão da possibilidade da dor, apesar do avanço científico e tecnológico na área de Odontologia, de forma que alguns pacientes usam termos como pavor, pânico e horror para descrever as sensações associadas ao tratamento. Nesse contexto, fica evidente a necessidade do relacionamento humanizado, com criação de vínculo, entre os envolvidos no procedimento, pois tal relação facilita o entendimento, a empatia e a alteridade no momento do tratamento. Trata-se de entender o paciente e tratar o indivíduo e não a sintomatologia, utilizando habilidades humanísticas nas relações interpessoais. Segundo Gaines APL, et al. (2021), desde a graduação, é fundamental desenvolver a competência de tratar o indivíduo e não sua patologia, treinando a capacidade de empatia, a inteligência emocional e a capacidade de comunicação.

O acolhimento como competência é adquirida desde a relação interpessoal acadêmica, visto que Rodrigues MP, et al. (2016) apontam que as relações conflituosas entre docentes e discentes têm efeitos negativos na formação do acadêmico. Em um ciclo, a conduta dos docentes reflete o que aprenderam na graduação. O trabalho de Perreira RGS, et al. (2020) reporta que os docentes identificam atitudes de alguns dos seus professores em suas práticas atuais. Portanto, segundo os autores, os docentes transmitem experiências vividas quando acadêmicos em suas práticas pedagógicas.

Os discursos legais e formais em torno da formação do profissional de Odontologia enfatizam a necessidade de formar um cirurgião-dentista humanista, mas a estrutura curricular vigente ainda está centrada na formação tecnicista. Esse paradigma pode ser mudado através do diálogo com outras áreas da ciência. Por exemplo, a Sociologia da Saúde é um ramo da Sociologia que estuda os fenômenos sociais do processo saúde-doença. A Sociologia da Saúde tende a influenciar positivamente a formação acadêmica do cirurgião-dentista, em conformidade com as Diretrizes Curriculares Nacionais (DCNs), que intencionam uma formação crítica e cidadã do profissional (FURLIN N, 2020).

Diante do exposto, conforme colocado por Silva RRD (2018), o bom relacionamento interpessoal entre os profissionais e com o paciente pode ser ferramenta útil na humanização do cuidado. Bem como a adequação da linguagem técnica para a melhor compreensão pelo paciente durante as orientações é uma conduta que pode encorajar o protagonismo do paciente, fechando o ciclo para promoção e proteção da saúde integral. 


\section{CONSIDERAÇÕES FINAIS}

No atendimento humanizado, o profissional da Odontologia precisa demonstrar preocupação com aspectos que vão além do atendimento técnico-especializado, da eficácia terapêutica e da qualidade do material utilizado. Os profissionais precisam ter uma perspectiva social e integral nos cuidados que prestam. O acolhimento adquire, portanto, importância crescente no processo do atendimento humanizado, na construção de uma relação de confiança baseada no vínculo, a qual é fundamental para a qualidade do cuidado em saúde. Dessa forma, o presente trabalho incentiva pesquisas sobre o acolhimento na Odontologia, principalmente, referentes às ferramentas e condutas que facilitem sua aplicabilidade, pois é importante oferecer um tratamento humanizado com visão social, boa comunicação e acolhimento para que os pacientes sejam mais colaborativos e os tratamentos tenham mais respostas positivas.

\section{AGRADECIMENTOS}

Os autores agradecem ao Centro Universitário Mario Pontes Jucá (UMJ, Maceió - AL) e à coordenação do curso de Odontologia (UMJ, Maceió - AL).

\section{REFERÊNCIAS}

1. CIANETTI S, et al. Dental fear/anxiety among children and adolescents. A systematic review. Eur J Paediatr Dent., 2017;18(2): 121-130.

2. COSTA G, et al. A integralidade, a partir do acolhimento, nas práticas de ensino clínico em uma faculdade de Odontologia. Arquivos em Odontologia, 2014; 50: 6370.

3. DARTIGUELONGUE JB, CAFIERO PJ. Communication in health care teams. Arch Argent Pediatr, 2021; 119(6): e589-e593.

4. DESCRITORES EM CIÉNCIAS DA SAÚDE. ID do descritor: DDCS050296. Disponível em: https://decs.bvsalud.org/ths/resource/?id=50296\&filter=ths_regid\&q=DDCS050296. Acessado em: 10 de fevereiro de 2022.

5. FURLIN N. A Sociologia na educação superior: sentidos produzidos nas narrativas de estudantes de Odontologia da Universidade Estadual de Maringá. Educação em Revista, 2020; 36: e219614.

6. GAINES A, et al. Humanização na Odontologia e práticas educativas na graduação. Enciclopédia Biosfera, 2021; 18(38): 733-747.

7. JACOMINE JC, et al. Saúde bucal e Pacientes com Necessidades Especiais: percepções de graduandos em Odontologia da FOB-USP. Revista da ABENO, 2018; 18(2): 45-54.

8. LOPES AS, et al. O acolhimento na Atenção Básica em saúde: relações de reciprocidade entre trabalhadores e usuários. Saúde em Debate, 2015; 39(104): 114-123.

9. LOPES GVDO, et al. Acolhimento: quando o usuário bate à porta. Revista Brasileira de Enfermagem, 2014; 67(1): 104-110.

10. MELO EA, et al. Indicators for dental appointment scheduling in primary health care: a national cross-sectional study. BMC Public Health, 2021; 21(1): 2234.

11. MILLEY KM, et al. Look who's talking now: Cancer in primary care on Twitter. An observational study. BJGP Open, 2021; 5(1): bjgpopen20X101134.

12. MINISTERIO DA SAÚDE. Secretaria de atenção à saúde. Departamento de atenção básica. Acolhimento à demanda espontânea. 1. ed. Brasília - DF: Brasil, 2013. Disponível em: https://bvsms.saude.gov.br/bvs/publicacoes/acolhimento_demanda_espontanea_cab28v1.pdf. Acessado em: 2 de fevereiro de 2022.

13. MINISTÉRIO DA SAÚDE. Secretaria de Atenção à Saúde. Núcleo Técnico da Política Nacional de Humanização. Humaniza SUS: Documento base para gestores e trabalhadores do SUS. 4. ed. Brasília - DF: Brasil, 2010. Disponível em: https://bvsms.saude.gov.br/bvs/publicacoes/humanizasus_documento_gestores_trabalhadores_sus.pdf. Acessado em: 2 de fevereiro de 2022.

14. MITRE SM, et al. Avanços e desafios do acolhimento na operacionalização e qualificação do Sistema Único de Saúde na Atenção Primária: um resgate da produção bibliográfica do Brasil. Ciência \& Saúde Coletiva, 2012; 17(8): 2071-2085.

15. MOTA L, et al. Humanização no atendimento odontológico: acolhimento da subjetividade dos pacientes atendidos por alunos de graduação nos campos de estágio. Revista brasileira de ciências da saúde, 2013; 16(4): 537-544.

16. PEREIRA RGS, et al. Saberes construídos na experiência e formação docente. Revista Docência do Ensino Superior, 2020; 10(e021722): 1-14.

17. RODRIGUES, MP, et al. Humanização: fragilidades, desafios e fortalezas em uma escola de Odontologia. Espaç. Saúde, 2015; 3(16): 27-38.

18. SCHNEIDER A, et al. Mental imagery in dentistry: Phenomenology and role in dental anxiety. J Anxiety Disord., 2018;58:3341.

19. SILVA KAR, DIAS AA. Compreensão sobre o atendimento humanizado em um ambulatório de Odontologia da marinha. Rev. bras. promoç. Saúde, 2019; 32: 1-10.

20. SILVA RM, et al. A visita domiciliar como prática pedagógica na formação em Odontologia. Rev. ABENO, 2017; 17(4): 87-98.

21. SILVA RRD. Humanização em saúde: A vivência em uma instituição de ensino. Dissertação. Universidade Federal de Minas Gerais, Faculdade de Odontologia, 2018; 69p.

22. SILVEIRA ER, et al. Estimated prevalence of dental fear in adults: A systematic review and meta-analysis. J Dent., 2021; 108: 103632. 